\title{
Methods for assessing risk factors to the health of highly qualified athletes
}

\author{
Davronbek Narzullaev $^{1^{*}}$, Alisher Tursunov ${ }^{1}$, Nodira Samigova ${ }^{1}$, Uktam Tursunov ${ }^{1}$, and \\ Kamoliddin Shadmanov ${ }^{1}$ \\ ${ }^{1}$ Tashkent pharmaceutical Institute, 45, Oybek street, Tashkent, 100015, Uzbekistan
}

\begin{abstract}
Currently, digital technologies are widely used both in the development of mass sports and sports of high achievements. Achievements in many sports disciplines are unthinkable without the use of various technical control schemes and computer processing. The development of sports focused on high world results requires the development and implementation of special technologies that allow planning to obtain extreme physiological and physical indicators of athletes by the time of the competition. The specificity of the tasks to be solved lies in the fact that each person is considered as a complex system, the state of which depends on many different factors. The manifestation of some of the factors can be traced according to the results of tests and competitions. Another part of the factors has a hidden, latent nature, individual for each person, and lies at the genetic level. The problem is how to find patterns that reflect the relationship between physiological, genetic and athletic performance. Coaches often make decisions based on their experience and intuition. As a rule, this is not always enough to achieve the goals set for the athletes. As one of the means of solving the problems described above, the use of methods of intellectual data analysis is proposed (IDA).
\end{abstract}

\section{Introduction}

The development of an information model for a comprehensive assessment and correction of health and sports form, prevention of the development of overtraining syndrome (OS), correction of vitamin D deficiency and associated deviations in the health of highly qualified athletes of the national teams of Uzbekistan is an urgent task today. The model should be based on the creation of a database that includes the results of clinical, laboratory, instrumental studies and sports tests, as well as risk factors for the athlete's health. All detected deviations must be corrected in the process of preparing athletes for the competition. Using the information model, based on the entered athlete's data, it will be possible to predict and eliminate the causes of OS development. The work identifies hidden patterns that affect health, fitness and OS development in an athlete. The main, auxiliary and hidden indicators for assessing the state of health and sports form will be divided, on the basis of which it will be possible to correct the identified deviations. The information model will make it possible to monitor in dynamics the state of health and sports form of athletes of the national teams

* Corresponding author: davr1960@mail.ru 
of Uzbekistan, which will allow achieving high results at competitions of the republican and world level.

\section{Materials and methods}

The work uses the following methods: intellectual data analysis [1-7], feature type conversion $[8,9]$, interval analysis methods $[10,11]$, a technique for searching for hidden patterns in the initial information $[12,13]$, questionnaires of athletes and coaches, sports tests, statistical methods.

According to [14] level of 25(OH) VD, IgE, of cytokines IFN- $\gamma$, IL-4 and TNF- $\alpha$ in blood serum was determined by IFA, respectively test systems DIAsource, Belgium, Human, Germany. Vector-Best, Russia. Parasitological methods included triple coproscopy using the ethyl acetate-formalin enrichment method.

As the initial information, a table of experimental data was used, obtained by questioning of 2520 athletes (various types of wrestling).

\section{Results}

The following risk factors for the health status and sports readiness of athletes can be distinguished $[15,16]$, which, in our opinion, are decisive in determining the causes of unstable performances of athletes:

- vitamin D deficiency;

- iron deficiency in the diet;

- impaired absorption of iron;

- physiological loss of hemoglobin in athletes;

- admission to training in highly qualified teams of persons with disabilities. Focuses of chronic infection, as well as previous rheumatism, inflammatory diseases of the heart, liver and kidneys, congenital defects and heart defects are especially dangerous;

- neglect of heredity, family diseases, early deaths in the family;

- training and competition in a painful state (acute diseases or exacerbations of chronic ones) or in case of insufficient recovery after them, which is accompanied by allergization of the body, a decrease in immunity, excessive stress of functions during exertion, a tendency to relapse, complications, overstrain, a drop in working capacity;

- inconsistency of morphological and functional characteristics with the chosen kind of sport, which increases the "price" of load and sports result for the organism, causing excessive stress of adaptation mechanisms;

- age discrepancy;

- irregular, non-rhythmic training;

- forced training (this is especially dangerous for young athletes who have not yet reached the proper level of development of adaptive mechanisms; during puberty);

- monotonous, highly specialized training, without switching, varying the conditions and means of preparation (especially in the early stages of sports specialization, and for qualified athletes - after reaching sports form);

- wrong combination of stress and rest, lack of conditions and means of recovery;

- long-term training against the background of underrecovery of the lost adaptation;

- frequent intense competitions and participation in them without the necessary preparation and against the background of underrecovery;

- lack of consideration of the age, gender, individual physical and psychological characteristics of the athlete; 
- psychological incompatibility with the trainer and participants;

- lack of psychological relief;

- improper use of pharmacological and other potent means of restoring and increasing sports performance;

- use of doping;

- frequent and massive weight loss;

- insufficient preliminary adaptation to training and competition in unfamiliar environmental conditions;

- inclusion of new sports in the competition program without sufficient preliminary study of their effect on the body (especially for women);

- unsatisfactory condition of training places, equipment, shoes and clothes of trainees;

- unfavorable weather conditions and environmental conditions;

- unbalanced, untimely nutrition, not meeting the requirements of the sport and the stage of preparation, poor quality of food and food preparation;

- lack of fortification;

- the use of alcohol, nicotine, drugs;

- shortcomings in the organization of classes and discipline;

- unfavorable living conditions;

- wrong combination of training with study or work;

- frequent stressful situations in sports, at work (study), at home and in the family;

- lack of general and sanitary culture;

- irregular and poor quality medical examination;

- lack of regular medical and medical pedagogical supervision;

- insufficient effectiveness of control methods, their discrepancy with the sport;

- inability of an athlete to conduct self-control, inadequacy of biomedical knowledge, inability to assess his condition and its change under the influence of various factors;

- untimely and poor-quality treatment of injuries and diseases in the process of playing sports;

- lack of hardening and means of increasing the specific and nonspecific resistance of the organism;

- insufficient and incorrect (without taking into account medical indicators) sanatorium treatment or its absence;

- lack of a well-founded prevention system;

- poor contact in the work of a doctor and a trainer: lack of proper biomedical knowledge of the trainer, his inability to use data from medical supervision, insufficient participation of a doctor in planning and correcting the training process;

- insufficient consideration of the peculiarities of their impact on the body;

- lack of special prophylaxis and protective devices;

- insufficient improvement of the special environment (water in the pool, the state of the tracks, etc.);

- repeated knockouts and knockdowns - falls in violation of the rules of admission after a traumatic brain injury.

Thus, 47 factors of the health status and sports readiness of athletes were identified.

As you know, a decrease in the performance of an athlete is associated with an iron deficiency and a decrease in the number of red blood cells in the blood. The essence of latent (latent) iron deficiency lies in the depletion of its transport and organ reserves, while the red blood indicators still remain within normal limits, which is most often the case in an athlete. Therefore, the task of increasing the physical working capacity and the ability to quickly 
restore the athlete's resources after extreme load becomes urgent. This is facilitated by pharmacotherapy with the help of special drugs that replenish iron deficiency.

The following reasons for the development of anemia can be distinguished:

- iron deficiency in the diet;

- violation of the absorption of iron;

- increased iron losses: with sweat, urine; due to increased bleeding of the gums; through the gastrointestinal tract; with helminthic invasion;

- low content of protein, vitamins C, B2, B12, folic acid in the diet;

- redistribution of protein, iron in favor of the formation of myoglobin myofibrils (working muscle hypertrophy), as a response to physical activity;

- hemolysis (destruction) of erythrocytes in blood vessels due to functional deterioration of erythrocytes; more often where the rheological properties of the blood are impaired, for example, the veins of the legs in runners;

- as a reaction to physical stress;

- physiological loss of hemoglobin in athletes.

In addition, a relative decrease in the concentration of hemoglobin in the blood of an athlete is possible due to an increase in the volume of circulating plasma.

Depletion of iron stores in the athlete's body leads to:

to a decrease in the level of physical performance due to:

- ergometric indicators,

- accumulation of lactic acid,

- changes in the body's gas gradients (oxygen and carbon dioxide);

- to overtraining.

At the beginning of the cycle of preparing an athlete for a competition, it is necessary to saturate the body with iron (in a course) to create its sufficient reserves. For women, during the "season", for the prevention of latent iron deficiency, two courses are given.

Therapeutic measures are carried out as anemia is detected, until the athlete's condition is normalized, both hemoglobin (at least $140 \mathrm{~g} / 1$ ) and "iron reserves" (ferrotin) are completely restored against the background of fortification and intake of anabolic herbal preparations, antioxidants. Treatment should begin as soon as iron deficiency is identified in the following order:

- Compensation for iron deficiency in blood and tissues (iron supplements).

- Restoration of the metabolism of erythrocytes and other blood cells (amino acids, anabolic agents, vitamins).

- Correction of the underlying causes of iron deficiency.

- Correction of lesions of the gastrointestinal tract, cardiovascular, hepatobiliary systems.

- Justified methods of treatment in the presence of visceropathy (damage to internal organs) is the complex use of essential phospholipids, metabolic agents that do not require an increased need for oxygen supply (mildronate, preductal), membrane stabilizers against the background of basic therapy with ferroreparations and antioxidants.

The duration of treatment depends on the initial level of hemoglobin and corresponds to 1-2 months. If a latent iron deficiency is detected, treatment is carried out according to the same scheme and with the same control for one month.

With a persisting source of blood loss, maintenance therapy continues. For example, with heavy periods at the end of menstruation, it is necessary to take iron preparations, amino acids, antioxidants for 7-10 days, every month, for 3-4 menstrual cycles. Control of hemoglobin in cyclic sports should be carried out monthly.

The therapeutic effect of most medicinal products (drugs) can be accompanied to one degree or another by side effects that may not develop in every athlete, but both the coach and the athlete who receive them need to know about them. 
Since drugs in most cases are elements alien to the human body and sometimes they lead to the development of undesirable or even dangerous phenomena. To replenish iron in the athlete's body, we used the "Fitoferon» medicinal collection developed at the Tashkent Pharmaceutical Institute. The pharmaco-toxicological properties of this drug collection were investigated, the effect of phytopheron on the activity of the liver in acute hepatitis was studied. It has been shown that it increases the body's resistance to infections, skin diseases, and has an adaptogenic effect. The results of the study of the chronic toxicity of the infusion of the collection "Fitoferon" showed that the studied drug in doses of $10 \mathrm{ml} / \mathrm{kg}$ and $25 \mathrm{ml} /$ $\mathrm{kg}$ was not toxic. When carrying out hematological and histological studies, the drug did not have a significant effect on the peripheral blood picture and the histomorphological picture of internal organs after prolonged administration.

Also, as a result of experiments, it was shown that taking $10 \mathrm{ml} / \mathrm{kg}$ or $25 \mathrm{ml} / \mathrm{kg}$ of phytopheron tincture for 1 month allows to restore peripheral hematopoietic elements and stimulates an increase in the number of erythrocytes.

In connection with the above, the level of iron in the athlete's blood was determined as the 48th risk factor.

Thus, there were created a table of experimental data (TED), consisting of 2520 lines (the number of examined sportsmen) and 48 columns (the sportsmen's health risk factors described above) and is the basis of the database. Methods of data mining were chosen as methods for assessing health risk factors for highly qualified sportsmen. For example, when solving the problem of automatic classification (dividing the set of objects (lines) of the TED into non-intersecting classes, the nearest neighbor algorithm was chosen [1]. In this case, all the signs (columns) of the TED must be quantitative (all mathematical operations are performed). The qualitative signs, there were applied methods of transforming the types of signs [8], allowing by digitization to translate these signs into quantitative. In the mathematical modeling of the issue under study were used the methods proposed in [9]. To determine the most important risk factors that have the greatest impact on health sportsman, the methods of decreasing the dimensionality of the initial attribute space for describing objects described in [4, 10-13] were applied. To solve the above problems, the intelligent data analysis system SITO-PC was used, developed by the authors of this article and implemented in an integrated development environment programmatically Delphi 10 Seattle software.

\section{Discussion}

Data Mining (DM) is a decision support process based on searching data for hidden templates (information templates). In this case, the accumulated information is automatically generalized to information that can be characterized as knowledge.

In general, the DM process consists of three stages:

- identifying templates;

- using the revealed templates to predict unknown values (predictive modeling);

- analysis of exceptions, designed to identify and interpret anomalies in the found templates.

Modern technologies of DM allow creating new knowledge, revealing hidden templates, predicting the future state of health and sports form of highly qualified athletes.

The purpose of creating an information model based on the DM methods is to identify hidden templates in databases describing the medical, physiological and sports parameters of athletes and, on their basis, to give a comprehensive assessment of health risk factors for athletes, as well as to develop procedures for eliminating the identified deficiencies in the training of highly qualified athletes. 
The use of the SITO-PC intelligent data analysis system in TED processing allowed us to conclude that the most important (informative) health risk factors for an athlete are vitamin $\mathrm{D}$ deficiency and low iron levels in the blood of the subjects. The Figure 1, presented below, gives the percentage diagram of the informativeness of health risk factors for an athlete when solving the problem of image recognition (classification in the presence of a training sample).

\section{Conclusion}

48 risk factors for the health of an athlete were identified and a database was created on their basis in accordance with [17]. In the developed database, each risk factor stated above is a separate field in the database. The program is implemented in the My SQL database management system, which allows users to create, edit and access information stored in the database, and is a standard for web servers that run under the Linux operating system. The proposed database is included in the information model, the purpose of which is to reveal hidden patterns in the databases describing the medical, physiological and sports parameters of athletes and, on their basis, to give a comprehensive assessment of the risk factors for the health status and sports form of athletes, as well as to develop procedures for eliminating the identified deficiencies in training highly qualified athletes.

To replenish iron in the athlete's body, the drug collection "Fitoferon» developed in Tashkent Pharmaceutical institute.

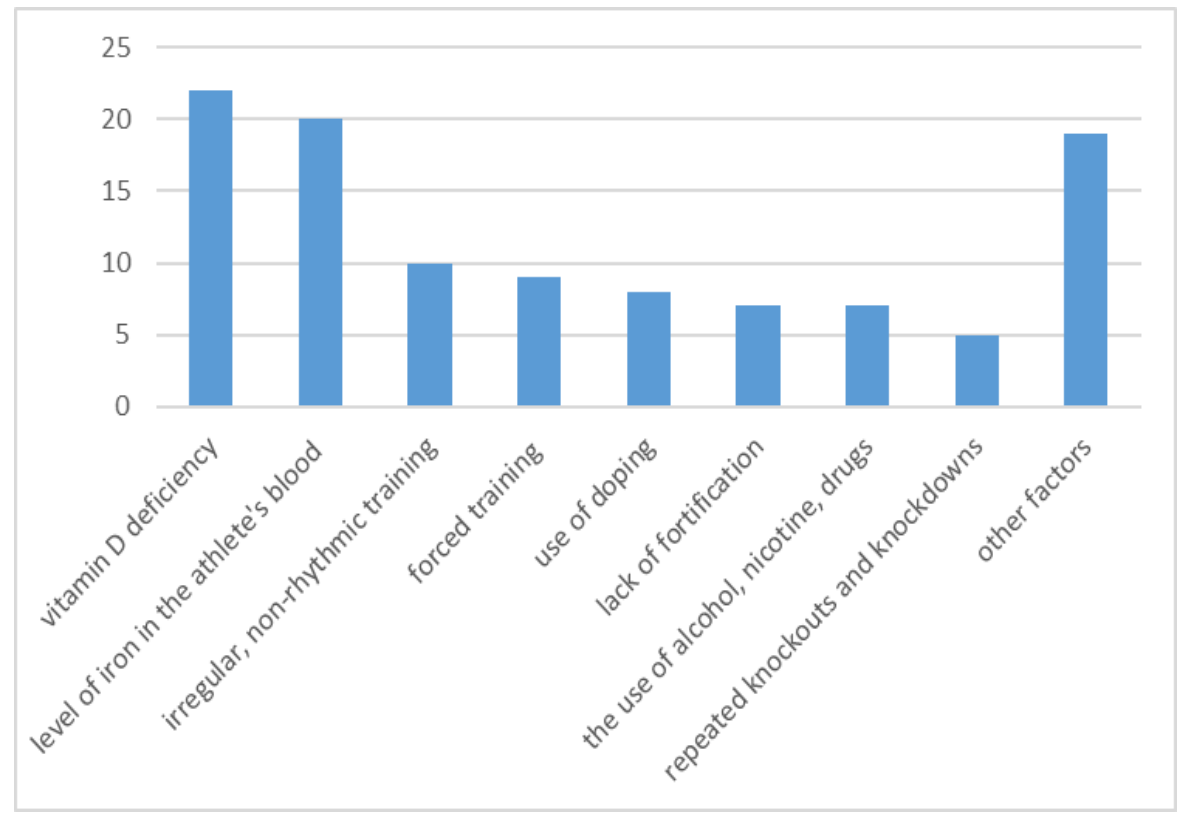

Fig. 1. Diagram of informativeness of health risk factors for an athlete - the result of work of the SITOPC data analysis system.

\section{References}

1. Ju.I. Zhuravljov, V.V. Rjazanov, O.V. Sen'ko, Recognition. Mathematical methods. Software system. Practical applications (Moscow: Fazis, 2006)

2. S.K. Fazilov, N.M. Mirzaev, S.S. Rajabov, O.N. Mirzaev, CEUR Workshop Proceedings 2098, 118-133 (2018) 
3. S. Fazilov, N. Mamatov, Journal of Physics: Conferens Series 1210(1) (2019)

4. S. Fazilov, N. Mamatov, A. Samijonov, A. Abdullaev, Journal of Physics: Conferens Series 1441(1) (2020)

5. Sh.Kh. Fazilov, N.M. Mirzaev, G.R. Mirzaeva, Modified Recognition Algorithms Based on the Construction of Models of Elementary Transformations, Procedia Computer Science pp 671-678 (2019)

6. Sh. Fazilov, N. Mamatov, N. Niyozmatova, International Journal of Recent Technology and Engineering 8, 2, 11, 3784-3786 (2019)

7. Sh. Fazilov, N. Mamatov, A. Samijonov, International Journal of Recent Technology and Engineering 8, 2, 11, 3790-3794 (2019)

8. D.Z. Narzullaev, B.A. Abdurakhmanov, A.S. Baydullaev, Sh.T. Ilyasov, K.K. Shadmanov, IOP Conference Series: Materials Science and Engineering 862 (5), 052065 (2020)

9. Kh. Sh. Ilhamov, D.Z. Narzullaev, Sh.T. Ilyasov, B.A. Abdurakhmanov, K.K. Shadmanov, IOP Conf. Series: Materials Science and Engineering 1047, 012021 (2021)

10. A.Kh. Nishanov, G.P. Djurayev, M.A. Khasanova, Compusoft 8, 6, 3158-3165 (2019)

11. A.Kh. Nishanov, G.P. Djurayev, M.A. Khasanova, Compusoft 9(6), 3725-3732 (2020)

12. A.Kh. Nishanov, B.B. Akbaraliev, G.P. Juraev, M.A. Khasanova, M.Kh. Maksudova, Z.F. Umarova, Journal of Cardiovascular Disease Research 11(2), 218-223 (2020)

13. A.Kh. Nishanov, B.B. Akbaraliev, B.S. Samandarov, O.K. Akhmedov, S.K. Tajibaev, Webology 17(1), 341-364 (2020)

14. J. Umarov, F. Kerimov, A. Toychiev, N. Davis, S. Osipova, Journal of Sports Medicine and Physical Fitness 59(12), 2058-2065 (2019)

15. D.Z. Narzullaev, K.K. Shadmanov, A.S. Baidullaev, E.E. Rajabov, A.T. Tursunov, IOP Conf. Series: Earth and Environmental Science 723 (2021), 032036 (2021)

16. N.D. Graevskaja, Sports and health 4 (1996)

17. D.Z. Narzullaev, D.F. Kerimov, Problems of physical culture and sports (ITA-PRESS, Tashkent: 2019) 